\title{
INFLUENCIA DA VARIABILIDADE CLIMÁTICA NA DENSIDADE DE MOSQUITOS VETORES NO LESTE DA AMAZÔNIA
}

\author{
SILVA, Rommel Benicio C. da - rbcsilva@yahoo.com.br \\ Universidade Federal do Pará / UFPA \\ VITORINO, Maria Isabel - vitorino@ufpa.br \\ Universidade Federal do Pará / UFPA
}

SOUSA, Adriano Marlisson Leão - adriano.souza@ufra.edu.br
Universidade Federal Rural da Amazônia / UFRA

SANTOS, Joyse Tatiane S. - joysetaty@gmail.com

Universidade Federal do Pará / UFPA

FERREIRA, Giovani Rezende B. - grezende2110@gmail.com
Universidade Federal do Pará / UFPA

GONÇALVES, Fabio Luiz Teixeira - fabio.goncalves@iag.usp.br Universidade de São Paulo / USP

SANTOS, Marcos Ronielly da Silva - mronielly@yahoo.com.br Universidade Federal do Pará / UFPA

\begin{abstract}
RESUMO: O clima na Amazônia tem apresentado mudanças naturais e principalmente antrópicas. Neste sentido este estudo objetiva entender a influência das mudanças climáticas na elevação da população de mosquitos na floresta primária da Amazônia oriental. Para isso, foram utilizados bancos de dados ambientais e biológicos coletados na Flona de Caxiuanã, como: Landsat 7 e 8, índices climáticos de IOS e MMA da NOAA, chuva, temperatura e umidade relativa do ar e biológicos do consórcio entre Museu Paraense Emilio Goeldi, FIOCRUZ, Instituto Evandro Chagas e Universidade Federal do Pará para um período de 1978 a 2017. Os resultados mostram que as mudanças no uso e cobertura da terra estão provocando alterações na variabilidade climática, promovendo a redução da precipitação e aumento de temperatura. No entanto, na área de floresta os padrões climáticos estão mais associados com a variabilidade natural. Além disso, as variações naturais do clima, através dos moduladores do Pacifico e do Atlântico indicam que as fases do ENOS determinam a variabilidade da precipitação anual. Apesar de 2005 ter ocorrido um extremo climático seco na Amazônia, mas a elevação da população de mosquitos parece estar relacionada com os efeitos da dinâmica atmosférica da escala de tempo.
\end{abstract}

PALAVRAS-ChaVe: Variabilidade Climática, Amazônia, Mosquitos, Desmatamento, Uso do solo.

INFLUENCE OF CLIMATE VARIABILITY IN THE DENSITY OF VECTOR MOSQUITOS IN EASTERN AMAZON

ABSTRACT: The climate in the Amazon has shown natural and mainly anthropic changes. In this sense, this study aims to understand the influence of climate change on the increase of the mosquito population in the primary forest of the eastern Amazon. For this, environmental and biological databases collected in the Caxiuanã Flona were used, such as: Landsat 7 and 8, NOAA IOS and MMA climatic indexes, rain, temperature and relative humidity and air from the consortium between Museu Paraense Emilio Goeldi, FIOCRUZ, Evandro Chagas Institute and Federal University of Pará for a period from 1978 to 2017. The results show that changes in land use and land cover are causing changes 
in climatic variability, promoting a reduction in precipitation and an increase in temperature. However, in the forest area, climatic patterns are more associated with natural variability. In addition, natural climate variations, through the Pacific and Atlantic modulators, indicate that the ENOS phases determine the variability of annual precipitation. Despite the fact that 2005 had an extreme dry climate in the Amazon, but the increase in the mosquito population seems to be related to the effects of the atmospheric dynamics of the time scale.

KEYWORDS: Climate Variability, Amazonia, Insects, Deforestation, Land use

\section{INFLUENCIA DE LA VARIABILIDAD CLIMÁTICA EN LA DENSIDAD DE LOS MOSQUITOS} VECTORIALES EN LA AMAZONIA ORIENTAL

RESUMEN: El clima en la Amazonía ha mostrado cambios naturales y principalmente antrópicos. En este sentido, este estudio tiene como objetivo comprender la influencia del cambio climático en el aumento de la población de mosquitos en el bosque primario del este de la Amazonía. Para esto, se utilizaron bases de datos ambientales y biológicas recolectadas en Caxiuanã Flona, tales como: Landsat 7 y 8 , índices climáticos NOAA IOS y MMA, lluvia, temperatura y humedad relativa y aire del consorcio entre Museu Paraense Emilio Goeldi , FIOCRUZ, Instituto Evandro Chagas y Universidad Federal de Pará por un período de 1978 a 2017. Los resultados muestran que los cambios en el uso de la tierra y la cobertura de la tierra están causando cambios en la variabilidad climática, promoviendo una reducción de la precipitación y un aumento de la temperatura. Sin embargo, en el área forestal, los patrones climáticos están más asociados con la variabilidad natural. Además, las variaciones climáticas naturales, a través de los moduladores del Pacífico y el Atlántico, indican que las fases ENOS determinan la variabilidad de la precipitación anual. A pesar de que 2005 tuvo un clima extremadamente seco en el Amazonas, pero el aumento de la población de mosquitos parece estar relacionado con los efectos de la dinámica atmosférica de la escala de tiempo.

PALABRAS CLAVE: variabilidad climática, Amazonía, insectos, deforestación, uso de la tierra

\section{INTRODUÇÃO}

O bioma Amazônia tem a maior floresta tropical úmida do globo terrestre, com maior biodiversidade do que as florestas úmidas da África e Ásia. Este bioma apresenta $10 \%$ das espécies conhecidas no mundo, o que constitui a maior coleção de plantas vivas e espécies animais (TURNER, 2001; SANTOS et al., 2011; WWF, 2017).

A variabilidade climática na região amazônica é principalmente modulada por fenômenos oceano-atmosfera que ocorrem sobre os oceanos Pacifico e Atlântico tropical, como o El Niño Oscilação Sul (ENOS) e o gradiente interhemisférico do Atlântico (FIGUEROA e NOBRE 1990; MARENGO, 1992; FISCH et al., 1998). A seca de 2005 apresentou anormalidade no gradiente interhemisférico do Atlântico e na oscilação sul no Pacifico, que ocasionou elevação na temperatura da superficie do mar e contribuiu para favorecer a redução das chuvas na Amazônia (MARENGO et al., 2008).

O regime das chuvas na Amazônia é marcado em toda sua extensão territorial, pelo ciclo anual do movimento migratório latitudinal da zona de convergência intertropical (ZCIT) sobre o atlântico equatorial (HASTENRATH e HELLER, 1977; NOBRE e SHUKLA, 1996 e RAO et al.,1996), que é o principal sistema que define a qualidade da estação úmida desta região. Os sistemas convectivos de mesoescala contribuem com $40 \%$ da precipitação, durante todo o ano (MOHR e ZIPSER, 1996; COHEN et al., 1995). Além desses sistemas, os 
vórtices ciclônicos de altos níveis (VCAN) e os distúrbios ondulatórios de leste (DOL) são os sistemas que atuam na época úmida da região.

Nas últimas décadas, a elevação das temperaturas globais, assim como o aumento do conteúdo do vapor d'água, que fortalece o ciclo hidrológico favorece as regiões úmidas a ficarem mais úmida e as regiões secas, mais secas, isso devido às tendências regionais e globais nos extremos de chuvas anuais nas últimas três décadas mostrarem que de âmbito global, tem ocorrido alteração na faixa anual de precipitação (CHOU et al., 2013).

No cenário de mudança no clima, pesquisas mostram alterações no tamanho de populações de mosquitos de acordo com as estações do ano que são expressivamente controladas pelas condições climáticas e pelas disponibilidades de alimentos e recursos ambientais, pois variam no mesmo ritmo (FORATTINI, 2002). Neste sentido, a temperatura do ar é a principal variável atmosférica responsável pela distribuição de doenças no Brasil, inclusive as negligenciadas, onde sua elevação faz com que mosquitos urbanos também sejam fortemente afetados por pequenos aumentos nesta variável para elevar o potencial da epidemia (PATZ et al., 1998; HOPP e FOLEY, 2003; LORENZ et al., 2017). Neste contexto, este trabalho visa analisar a influência das mudanças climáticas em suas componentes natural e antrópica na elevação de densidade da população de mosquitos na região de floresta na Amazônia oriental.

\section{MATERIAL E MÉTODOS}

A área de estudo compreende grande parte da região centro norte do Estado do Pará, representado pelos limites do quadrado, conforme pode ser visto na figura 1. Esta região é composta por uma diversidade de uso e cobertura do solo que inclui uma Unidade de Conservação (UC), denominada Flona de Caxiuanã, áreas urbanas, estradas, vegetação e vasta hidrografia.

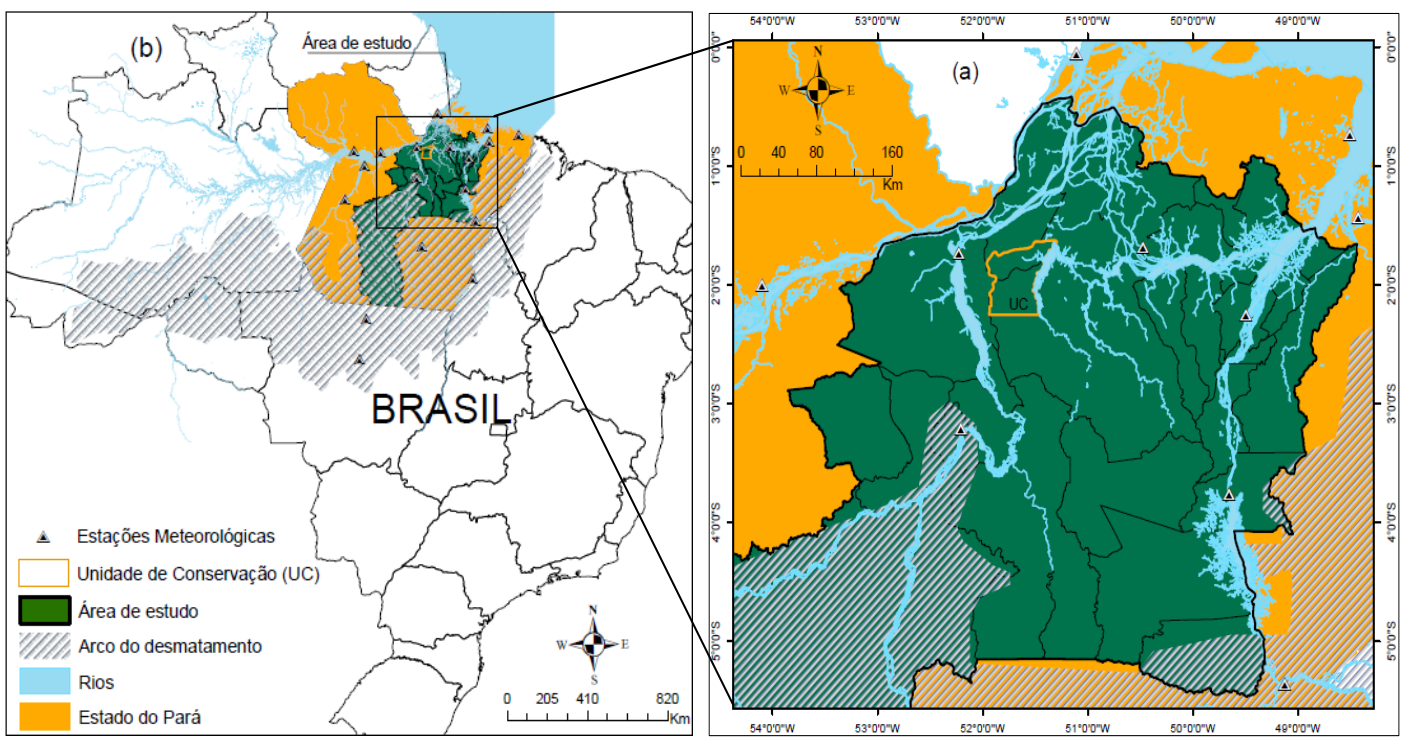

Figura 1 - Mapa de localização da área de estudo (verde) a nível nacional e estadual, indicando a área da unidade de conservação e região no entorno (a), e as estações meteorológicas ( $\square$ ) nos municípios de abrangência (b). 
Inicialmente para a obtenção das características ambientais da superfície foram utilizados os dados do projeto TerraClass descritos nas órbitas-ponto do satélite Landsat 5 (sensor TM) e Landsat 8 (sensor OLI), disponível no site do Instituto Nacional de Pesquisas Espaciais (INPE). Estes dados foram usados com o intuito de obter um diagnóstico espacial qualitativo do uso e cobertura do solo para uma década, contida na série temporal dos dados ambientais, visando identificar marcadas mudanças da superfície. Para isso, foram usadas um total de 10 imagens para cada ano de 2004 e de 2014.

Para caracterização do ambiente atmosférico na escala de clima foram utilizados os dados mensais dos índices climáticos dos oceanos Pacifico que é o Índice da Oscilação Sul (IOS) e do Atlântico tropical que é o Índice do Modo Meridional do Atlântico (MMA), que representam a variabilidade dos fenômenos oceano-atmosfera do ENOS e do Dipolo do Atlântico, através das fases do IOS e do MMA para o período de 1978 a 2017. Estes dados estão disponíveis no site da National Oceanic and Atmospheric Administration (NOAA) (http://www.cpc.ncep.noaa.gov/data/indices/). Para análise desses dados foram aplicados a média móvel de 12 meses e foi considerado os eventos extremos com índices acima +2 e abaixo -2 . Isto tem por finalidade identificar e caracterizar as condições climáticas a partir dos extremos das fases positiva (IOS e MMA) e negativa (MMA e IOS) dos índices que respondem pelo aumento e diminuição da precipitação na região de estudo, respectivamente.

Ainda no contexto climático foram usados os dados meteorológicos mensais, obtidos junto ao banco de dados meteorológicos (BDMET), disponível no site do Instituto Nacional de Meteorologia (INMET) para a precipitação (PRP $\mathrm{mm}$ ) ou chuva, temperatura média $\left(\operatorname{Tar}^{\circ}{ }^{\circ} \mathrm{C}\right.$ ) e a umidade relativa (UR \%) do ar, observadas nas 15 estações meteorológicas, durante 1987 a 2017, localizadas em geral nas áreas urbanas dos seguintes municípios: Altamira, Belém, Belterra, Breves, Cametá, Conceição do Araguaia, Itaituba, Marabá, Monte Alegre, Óbidos, Porto de Móz, São Félix do Xingú, Soure, Tracuateua e Tucuruí. Além desses, ainda foram utilizados os dados dos municípios de Gurupá, Curralinho, Limoeiro do Ajurú, S. S. B. Vista, Anapú, Bagre, Brasil Novo, Breu Branco, Igarapé Miri, Itupiranga, Medicilândia, Melgaço, Mocajuba, Mojú, Muaná, N. Repartimento, O. do Pará, Pacajá, Portel, Sen. J. Porfírio e Vitória do Xingú, todos no estado do Pará e que formam a grande área deste estudo (Figura 1b).

Além da fonte do INMET foram usados os dados horários da torre micrometeorológica pertencente ao Programa LBA (The Large Scale BiosphereAtmosphere Program in Amazonia) que está instalada na área da Estação Científica Ferreira Penna (ECFPn) dentro da Unidade de Conservação (UC), conhecida como FLONA de Caxiuanã, área de floresta primária. Esta torre de monitoramento atmosférico do Programa LBA tem 52 metros de altura, equipada por diversos aparelhos que medem e registram informações das componentes atmosféricas, onde foram utilizadas apenas a Tar, UR e a PRP, durante o período de 2000 a 2017. Esses dados apresentaram algumas falhas, como a descontinuidade de medidas e de valores espúrios para o período de abril de 2014 a dezembro de 2015 e em março de 2015, com chuva acima de $1230 \mathrm{~mm}$. Para o preenchimento de falhas e a correção de valores na escala mensal foi utilizado dados das estações meteorológicas da rede do INMET mais próxima da torre, utilizando o método de Regressão Linear Múltipla, conforme descrito em Mello et al., 2017. 
Os dados climáticos e meteorológicos de PRP, Tar e UR das fontes do INMET e do LBA foram processados conjuntamente de modo a apresentarem a mesma escala espacial e temporal. Com o intuito de obter-se a variabilidade espacial e temporal da área de estudo, foram obtidas através da média anual e anomalia da PRP, a média espacial climatológica (1978 a 2017) e a média decadal para os períodos de: 1978-1987, 1988-1997, 1998-2007 e 2008-2017. Além desses, foram selecionados os eventos de extremos climáticos a partir das anomalias negativas ou positivas de chuvas, relacionadas com os indicadores climáticos. A análise dos padrões médios climáticos e por década das variáveis meteorológicas podem sugerir os padrões espaço-temporal associados aos fenômenos naturais e/ou antrópicos.

A aquisição das informações, referentes à densidade de mosquitos capturados na UC da Flona de Caxiuanã, foram obtidas a partir do consorcio entre as instituições de pesquisas, Museu Paraense Emilio Goeldi, FIOCRUZ, Instituto Evandro Chagas e Universidade Federal do Pará. A coleta e captura dos mosquitos ocorreram em cinco campanhas, com duração de 6 dias cada, durante os meses de julho, setembro e dezembro de 2005, e fevereiro e abril de 2006, totalizando 33.122 indivíduos capturados em todas as campanhas. Para a captura dos espécimes foi aplicada a metodologia do atrativo humano (AH) e armadilhas luminosas, conhecidas como CDC (Control Disease Center) e estão detalhadas nos estudos de Pinto (2007), Pinto et al. (2009) e Confalonieri e Neto (2012). Assim, a análise horária da densidade de mosquitos com as variáveis meteorológicas na região da UC no período de campanha visa entender a influência das mudanças climáticas, devido as variações ambientais naturais e antrópicas, combinadas com uma elevada densidade de mosquitos.

\section{RESULTADO E DISCUSSÃO}

\subsection{ANÁlISE DA MUDANÇA DE USO DO SOLO}

Grandes mudanças no uso do solo na região de fronteira entre os municípios localizados no centro sul do mapa (figura 2), ou seja, a sul da área da UC. Esta mudança pode estar relacionada com a intensificação do desmatamento para exploração madeireira e a introdução da pecuária, com o aumento de áreas de pastagem e urbana. Ainda se observa mudanças no solo a noroeste da UC, com aumento na retirada da floresta. O desmatamento na região de estudo apresenta um cenário onde modificações no uso e cobertura da terra está se evidenciando, principalmente pelas ações humanas que adentram na floresta por todos os lados, principalmente pela parte sul da área de estudo. 


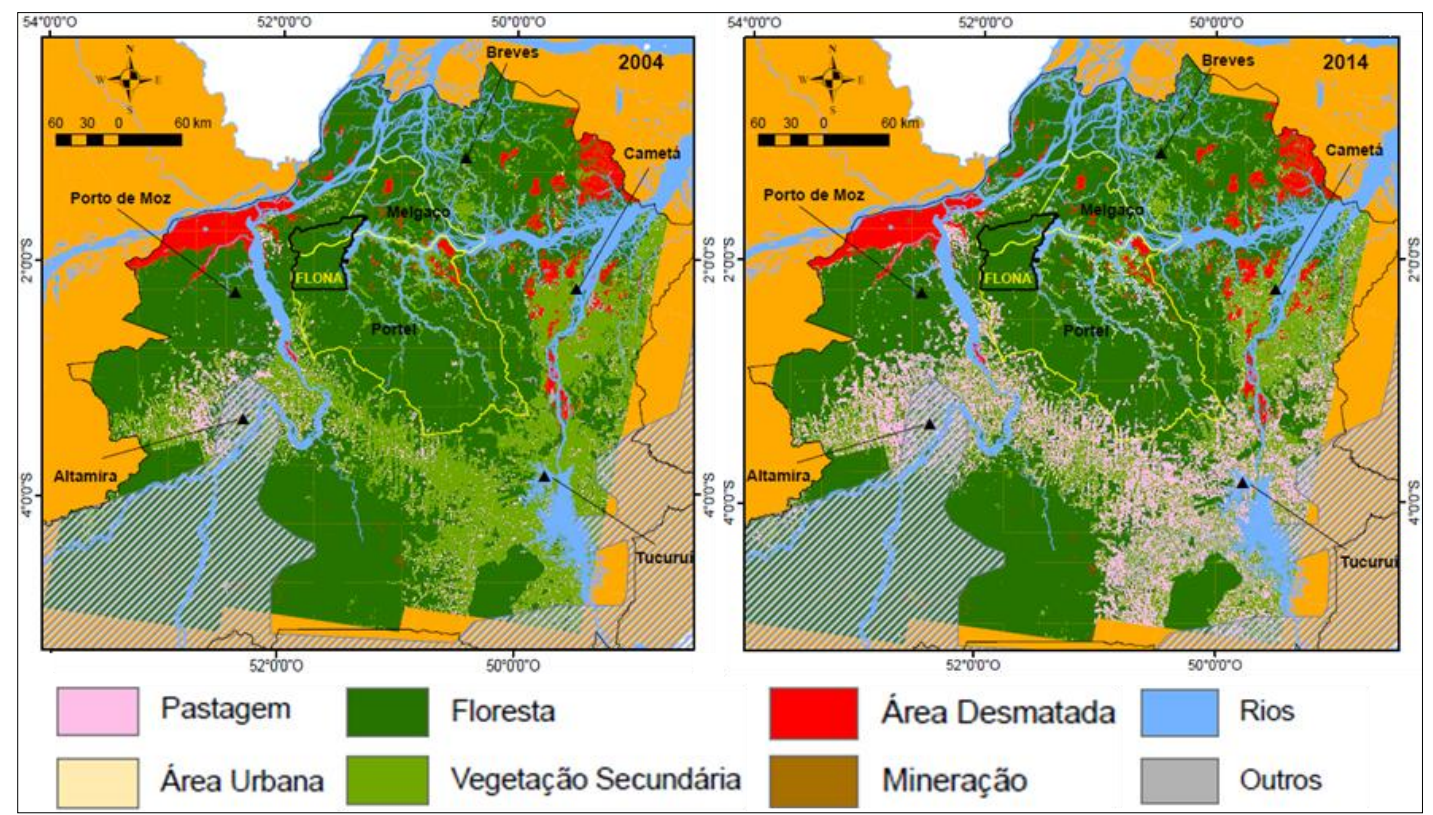

Figura 2 - Variação do uso e cobertura do solo na região de estudo, durante o período de a) 2004 e b) 2014.

Nestas análises, observa-se que o município de Portel teve uma intensificação expressiva de desmatamento na sua parte sul. O município de Altamira tem as margens de sua região metropolitana, um completo cenário de mudança do uso do solo, através do avanço de atividades agrícolas, ações de garimpo e invasões, inserindo este município, conjuntamente com Tucuruí, dentro da área do arco do desmatamento (CARVALHO et al., 2016).

A retirada da floresta para a plantação de pastagens e lavouras faz com que estes novos cenários deixem de liberar água para a atmosfera, por absorver menos energia do que a vegetação original (florestas primárias), contribuindo significativamente para a redução das chuvas nesta área e acarretando elevação na temperatura do ar (NEPSTAD et al., 1999).

\subsection{ANÁLISE CLIMÁTICA TEMPO-ESPAÇO}

Inicialmente, visto de modo geral através da média móvel (figura 3) uma tendência de um padrão dos índices climáticos MMA e IOS, com variabilidades multidecadal e interanual mais negativo nas duas primeiras, e mais positivo nas duas últimas décadas. No periodo de 1978 a 1997 o IOS negativo (El Niño) apresenta os maiores indices observados, com relação toda a série. Por outro lado, o MMA negativo provoca o deslocamento da ZCIT mais ao sul do equador, causando mais chuvas na área de estudo.

Assim, o somatório dessas duas forçantes moduladoras do clima podem levar a consequência de uma resultante de anomalias negativas de chuvas na região, pois o IOS parece indicar mais energia do que o MMA. No período de 1988 a 2017, se percebe que a maior frequencia positiva dos índices MMA e IOS. No entanto, com o MMA desfavorável, o IOS positivo é muito frequente favorável a La Niña, portanto contribuindo para o aumento de chuvas. Isto 
porque o sinal do IOS do pacifico é mais duradouro do que as variações do altântico, desse modo ele influencia por mais tempo as circulações atmosférica.

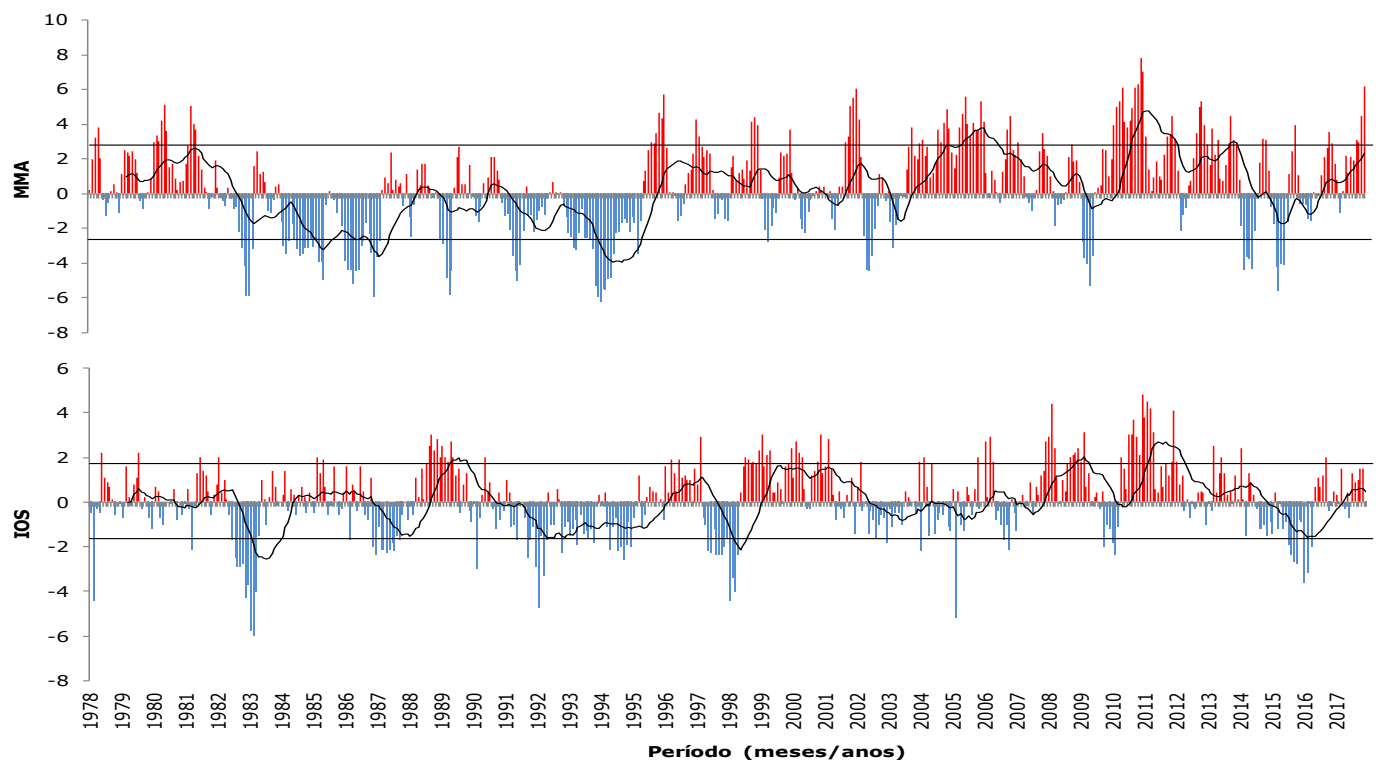

Figura 3 - Distribuição mensal dos índices climáticos MMA (a) e IOS (b), durante o período de 1978 a 2017. As linhas pretas curvas representam a média móvel de 12 meses, as horizontais indicam os limiares dos extremos climáticos e as verticais divisão decadal.

Segundo Gonzalez et al. (2013) a relação entre as bacias do pacifico e atlântico equatorial apresentam maior significância, quando o El Niño (IOS -/+) se associa ao atlântico (MMA -/+) promovendo a diminuição/aumento das chuvas no leste da Amazônia. Assim, este estudo apresenta concordância com os autores, a nível de dois períodos de duas décadas para a variação dos índices IOS e MMA.

Através das anomalias de precipitação anual (figura 4) os efeitos causados pelos fenômenos climáticos representados pelo IOS e pelo MMA onde o índice do pacifico, o IOS, parece governar a variabilidade das chuvas anuais na região de estudo. Pode ser notado que a precipitação indica uma tendência ao aumento de chuvas com dois períodos bem definido de 20 anos pelas anomalias negativas de chuvas de 1987 a 1997 e positivas 1998 a 2017. 


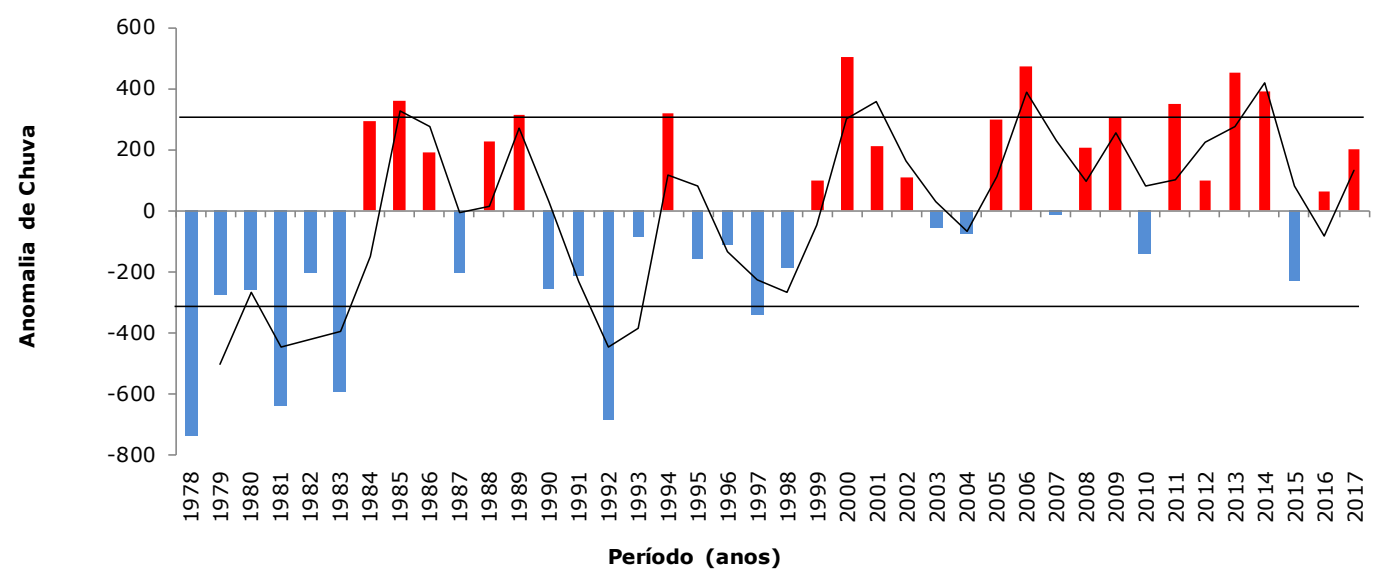

Figura 4 - Anomalia anual de chuva para a média da área de estudo, durante 1978 a 2017. As linhas pretas curva de chuva é a média móvel anual, as horizontais são as faixas do limiar das anomalias para extremos de chuvas (cores vermelha chuvas acima da média e azul abaixo da média climática) e as verticais a divisão decadal.

A variabilidade interanual dos indices climáticos combinados com as anomalias de chuvas sugere dois períodos de 20 anos bem definidos, uma com dominância de extremos secos (1978-1997) e a outra de extremos úmidos (1997-2017). Os extremos climáticos proporcionam impactos significativos nas variáveis atmosféricas, tanto sobre as cidades quanto sobre as florestas, promovendo alteração nos níveis de temperatura do ar, umidade relativa e precipitação.

É importante comentar as características climáticas das duas maiores secas observadas na Amazônia nas últimas décadas, a de 2005 e a de 2010. Nestas secas 2005/2010, os indicadores climáticos se mostram positivo para o MMA e negativo para o IOS com magnitudes diferentes, e com anomalias positiva/negativa de chuvas, respectivamente. A seca de 2005 o El Niño não interferiu nas chuvas, porém apesar do atlântico está desfavorável, observa-se chuvas acima do normal. No entanto, na seca de 2010 o El Niño combinado com a fase desfavorável do Atlântico intensificou os efeitos da redução das chuvas na região (Figuras 3 e 4). Estes resultados estão de acordo com os obtidos por Marengo et al., (2008; 2011).

As variabilidades multidecadais da chuva (tabela 1), uma no período de 1978 a 1997 com 6 eventos de extremos climáticos secos e 5 úmidos em 1997 a 2014 na área de estudo. Percebe-se que para todos os extremos secos o IOS representa $83 \%$ e o MMA $27 \%$, e para os úmidos, $80 \%$ de IOS e para $20 \%$ do MMA. Dessa maneira, pode ser notado que o IOS apresenta maior atuação nos extremos climáticos para a região. 
Tabela 1 - Relação dos extremos climáticos a partir das anomalias de chuva, associado ao valor médio anual dos índices IOS e MMA, durante 1978 a 2017.

\begin{tabular}{lccll} 
& aChuva & MMA & IOS & Indicador \\
\hline 1978 & -735.09 & 0.72 & -0.12 & MMA/IOS \\
1981 & -640.79 & 1.84 & 0.41 & MMA \\
1983 & -593.97 & -0.30 & -1.26 & IOS \\
1985 & 362.25 & -1.91 & 0.48 & MMA/IOS \\
1992 & -685.94 & -0.50 & -1.37 & IOS \\
1997 & -342.56 & 1.08 & -1.21 & IOS \\
2000 & 504.47 & -0.45 & 1.39 & MMA/IOS \\
2006 & 474.45 & 1.73 & 0.02 & IOS \\
2011 & 348.73 & 2.48 & 2.31 & IOS \\
2013 & 449.45 & 2.51 & 0.75 & IOS \\
2014 & 389.89 & -0.86 & -0.22 & MMA \\
\hline
\end{tabular}

A variabilidade mensal das variáveis meteorológicas nas áreas urbana e de floresta (figura 5) que evidencia que os ambientes respondem de modo diferente na floresta com relação a urbana. A chuva e a umidade do ar em um ambiente de floresta, apresenta elevações expressivas, em relação as áreas urbanas, apenas a temperatura do ar apresenta decréscimo. Isto sugere que as temperaturas do ar mais acentuadas na área urbana estão relacionadas com a resposta do balanço de radiação e energia em superfície sem cobertura vegetal.
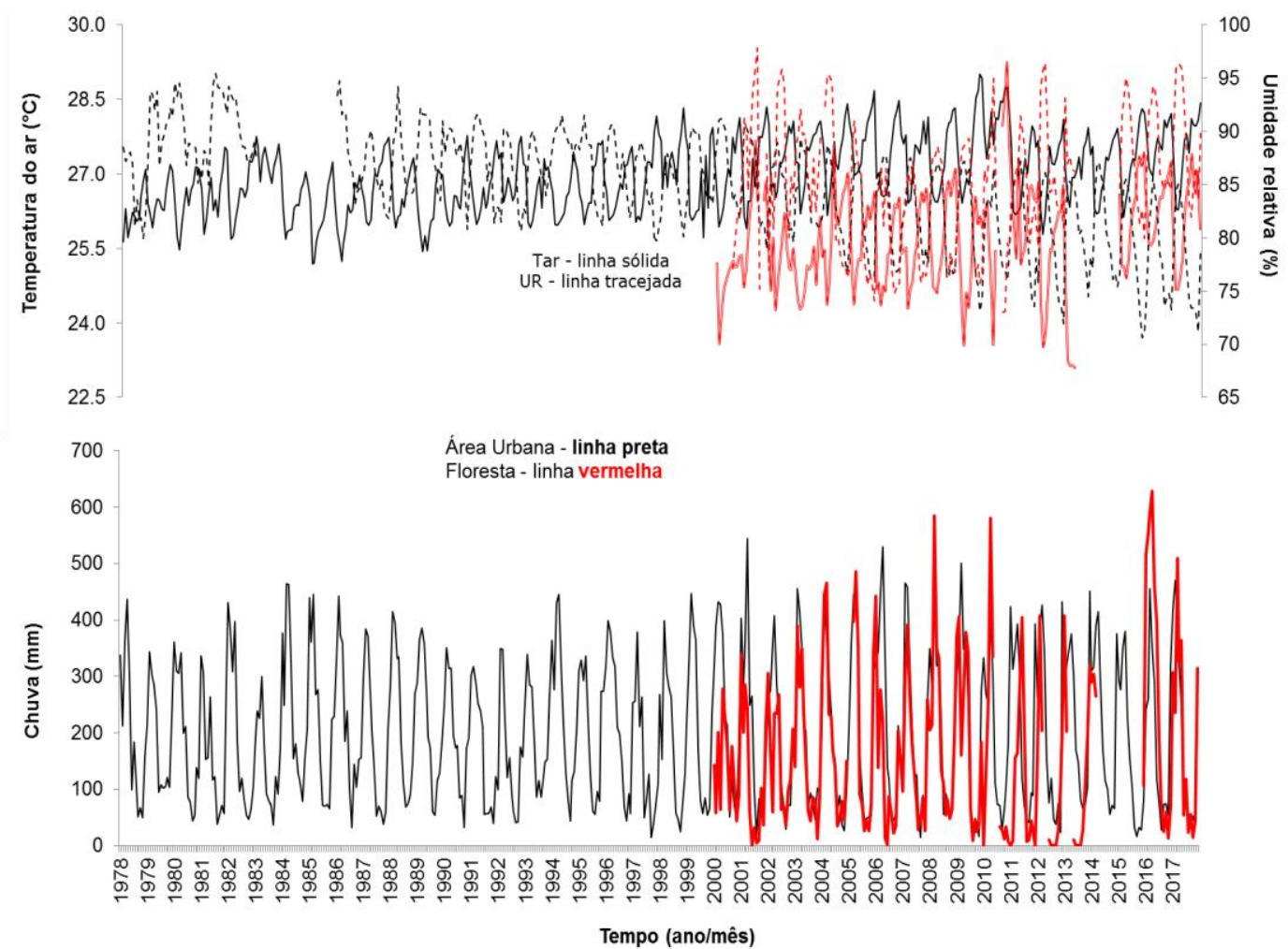

Figura 5 - Distribuição mensal da chuva, da temperatura média e da umidade relativa do ar para as áreas urbanas (linha preta) e de floresta (linha vermelha), durante os periodos de 1978 a 2017 e 2000 a 2017, respectivamente. 
Vale ressaltar que o clima da região de estudo no período das campanhas de coleta dos dados biológicos (2005 e 2006) apresentam chuvas acima da normalidade na área urbana (figura 4), ou seja, no entorno da UC. Mas, quando se compara as chuvas nas áreas de floresta e urbana estas são similares, exceto em 2006 que a área urbana apresenta maior quantitativo. No entanto, as temperaturas foram mais elevadas, com menores índices de umidade do ar para área urbana, com o inverso para a floresta (figura 5). O estudo de Nepstad et al. (1999) observa que a variáveis atmosféricas para regiões de floresta e urbana respondem de modo similar ao presente estudo.

O padrão médio climatológico (figura 6), com os maiores valores de chuva e de temperatura do ar a nordeste e redução a sudoeste da região, onde se localizam os maiores corpos hídricos, juntamente com a proximidade do oceano. A umidade relativa apresenta pequena expressão, apenas com máximos (mínimos) valores nos extremos, nordeste e sudoeste (sudeste), onde se concentram as maiores (menores) fontes de umidade (arco desmatamento), a oceânica e a floresta, respectivamente.

Ainda pode ser visto que a área da UC e em seu entorno (figura 6) sugere uma preservação natural, devido a própria dinâmica da floresta e a proximidade de transporte de vapor dos corpos hídricos a nordeste. Segundo Nobre (2014) a floresta produz a sua própria chuva que permite o equilíbrio dos níveis de temperatura, conforme a conservação de sua estrutura vegetal.

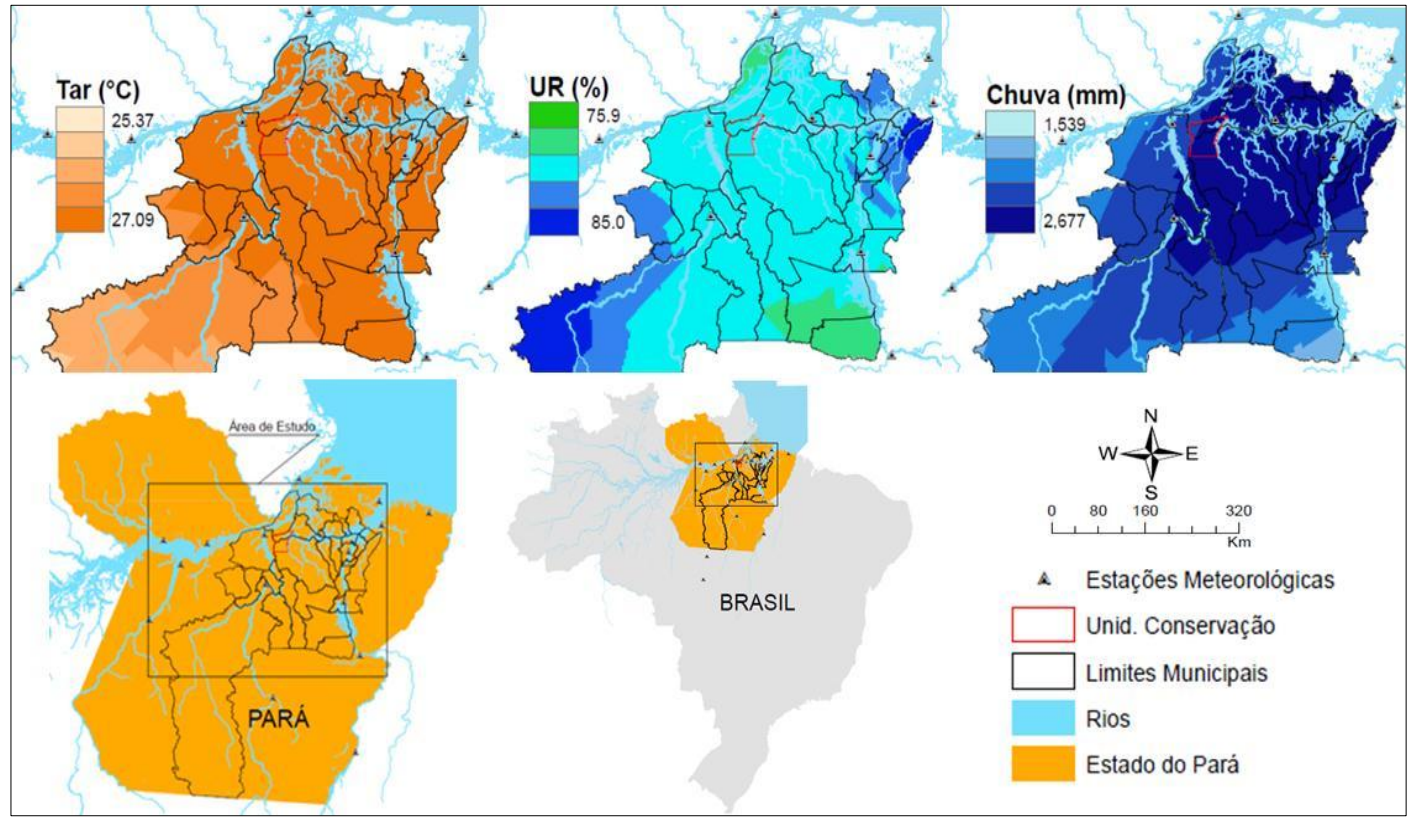

Figura 6 - Climatologia espacial da temperatura do ar, da umidade relativa do ar e da chuva para a região de estudo, com efeito na unidade de conservação (UC), durante o período de 1978 a 2017.

A análise decadal da temperatura e da umidade relativa do ar indicam os maiores valores no geral, exceto para a região da UC, durante as primeira e segunda década de 1978 a 1987 e 1988 a 1997. Para as duas últimas décadas, se observa que o setor centro norte revelam os maiores valores e com leve diminuição a sudoeste e sudeste da região. Os maiores acumulados de chuvas se localizam nas áreas de fontes hídricas, a nordeste da região. No entanto, 
pode ser notado que nas duas primeiras décadas as chuvas são melhor distribuidas, indicando alterações no setor centro sul da região, a partir da duas últimas décadas (figura 7).

Torna interessante combinar os mapas da mudança e uso do solo (figura 2), com as fases multidecadais dos indicadores climáticos (figura 3) e as variaveis meteorológicas em escala decadal (figura 7) para uma visão acoplada dos efeitos naturais e antrópicos. Dessa forma, percebe-se o efeito antrópico da mudança do solo (Figura 2 b) no centro sul, a partir da redução da umidade e do aumento da temperatura, durante a última década de 2008 a 2017, quando foi observado a mudança na cobertura vegetal. Além disso, fica notório a influencia da variabilidade climática natural, durante a segunda década e nas duas ultimas, mas com interferência antrópica.

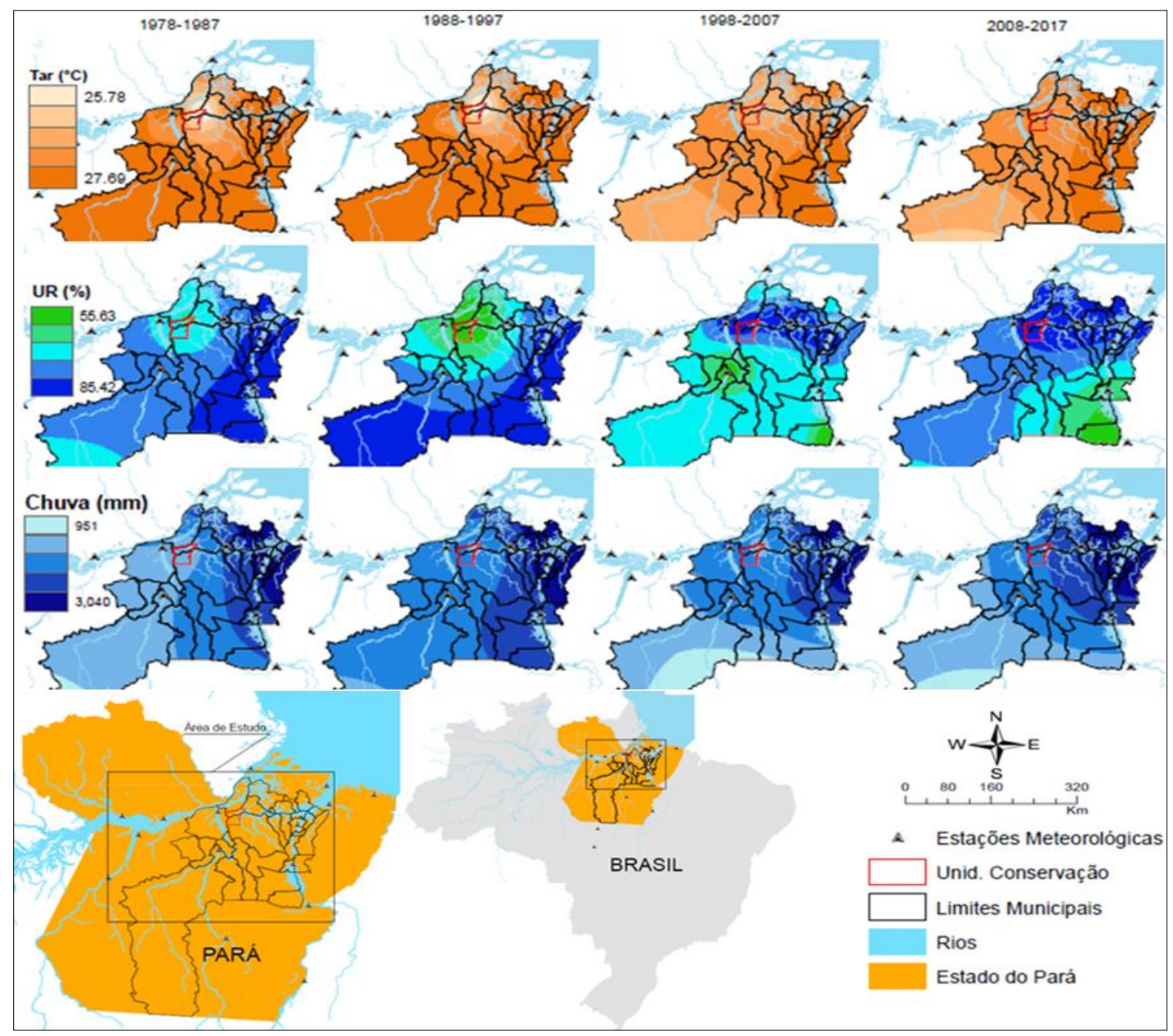

Figura 7 - Variabilidade espacial por década (1978-1987;1988-1997; 1998-2007; 20082017) para a temperatura do ar (Tar), a umidade relativa do ar (UR) e a chuva para a região de estudo, com efeito na unidade de conservação (UC).

\subsection{DENSIDADE DE MOSQUITOS E O CLIMA}

A distribuição geral dos mosquitos (Tabela 2), conforme o gênero e a quantidade dos espécimes capturados demonstra o quanto a densidade de cada gênero (mosquito) se comporta em relação ao período do ano. Pode ser 
observado que os espécimes dos gêneros Culex (transmissores da mielite, encefalite e zika), Haemagogus e Sabethes (transmissores da febre amarela), Coquillettidia, Ochlerotatus, Limatus, Wyeomyia, Phoniomyia (transmissores de arboviroses), Anopheles (transmissores da malária) e Lutzomyia (transmissores da Leishmaniose) foram capturados em todas as cinco campanhas, os demais foram ausentes em, no mínimo, uma campanha. Além disso, se observa que o mês de setembro de 2005 apresenta a maior densidade de mosquito, seguido por fevereiro de 2006.

Tabela 2 - Quantidade total de espécimes capturados, classificados por gênero, período do ano e totalidade.

\begin{tabular}{|c|c|c|c|c|c|c|}
\hline & & & & & & \multirow[b]{3}{*}{ Total } \\
\hline \multirow[b]{2}{*}{ Gênero } & \multicolumn{5}{|c|}{ Período } & \\
\hline & jul/05 & Set-05 & Dez-05 & Fev-06 & Abr-06 & \\
\hline Culex & 3592 & 10346 & 3886 & 6799 & 3674 & 28297 \\
\hline Lutzomyia & 186 & 525 & 239 & 219 & 180 & 1349 \\
\hline Coquillettidia & 340 & 339 & 10 & 519 & 12 & 1220 \\
\hline Haemagogus & 168 & 61 & 23 & 631 & 115 & 998 \\
\hline Sabethes & 129 & 41 & 51 & 151 & 125 & 497 \\
\hline Wyeomyia & 54 & 29 & 87 & 104 & 71 & 345 \\
\hline Ochlerotatus & 47 & 8 & 2 & 56 & 99 & 212 \\
\hline Limatus & 9 & 12 & 9 & 45 & 26 & 101 \\
\hline Anopheles & 16 & 1 & 1 & 15 & 6 & 39 \\
\hline Uranotaenia & 6 & 15 & 2 & & 1 & 24 \\
\hline Phoniomyia & 3 & 2 & 2 & 6 & 1 & 14 \\
\hline Mansonia & 7 & 5 & 2 & & & 14 \\
\hline Psorophora & & & & 1 & 2 & 3 \\
\hline Chagasia & 2 & & & 1 & & 3 \\
\hline Orthopodomyia & & 2 & 1 & & & 3 \\
\hline Thricoprosopon & & 1 & & & 2 & 3 \\
\hline Total & 4559 & 11387 & 4315 & 8547 & 4314 & 33122 \\
\hline
\end{tabular}

A quantidade de mosquitos e as condições de tempo e clima no período de campanha (figura 8) sugerem que a maior densidade de mosquitos parece oscilar conforme a variação da temperatura do ar, apresentando elevação em sua densidade, logo após um aumento significativo, seguido de redução na temperatura do ar (figura 8a). A variação da umidade relativa do ar (figura 8b) é inversa da temperatura, embora não houve registro de eventos de chuva (figura 8c), sugere que este seja uma das causas da elevação expressiva no número de indivíduos capturados nesta campanha. 


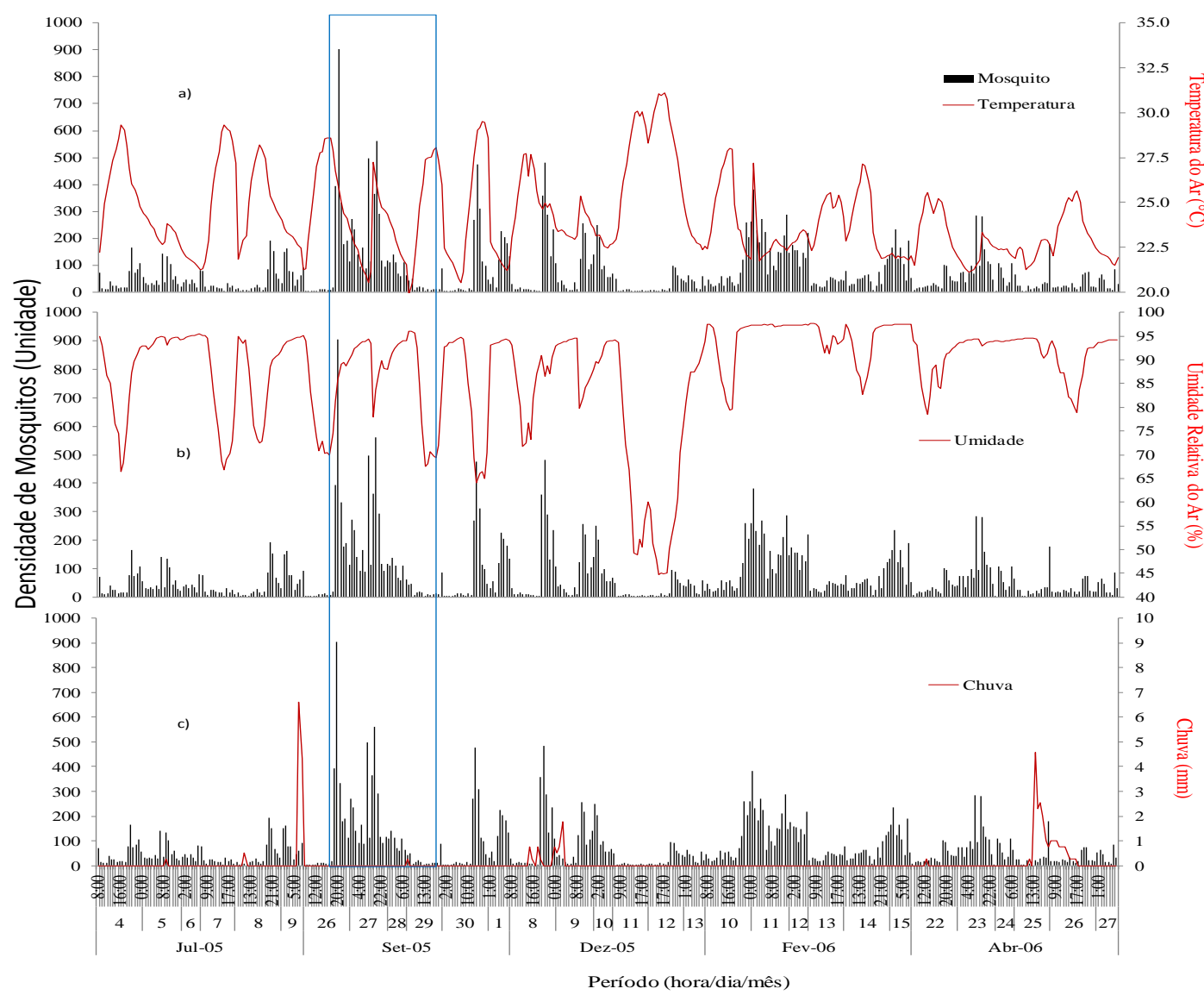

Figura 8 - Distribuição horária da densidade de mosquitos (preto) e das variáveis meteorológicas (linha vermelha) da temperatura do ar (a), umidade relativa do ar (b) e da PRP (c) na região da Unidade de Conservação (UC), com destaque no período de maior quantidade de espécimes (quadrícula azul), durante as campanhas de 2005 e 2006.

A oscilação da densidade dos mosquitos pode estar sendo fortemente influenciada pelos níveis de temperatura do ar e de chuva. Os mosquitos que possuem gigantescas modificações em sua densidade (mosquitos explosivos), se desenvolvem em coleções líquidas de caráter transitório ou temporário. A espécie Culex portesi se desenvolvem em habitat terrestres de caráter permanente ou semipermanente, o que exclui esta espécie do contexto explosivo, fortalecendo a ideia de que esta espécie respondeu significativamente à variabilidade climática do ambiente (FORATTINI, 2002).

\section{CONCLUSÃO}

A abordagem deste estudo contempla a influência dos efeitos das mudanças climáticas na elevação de densidade da população de mosquitos na região de floresta na Amazônia oriental. Para isso foram obtidas as seguintes conclusões:

As mudanças no uso e cobertura do solo indicam alterações nas variáveis climáticas, principalmente na década de 2008 a 2017; 
A variabilidade climática da precipitação sofre maior influencia do indicador do pacifico, o IOS, do que do atlântico tropical, o MMA, indicando dois períodos multidecadais, com redução e aumento de precipitação anual;

As mudanças climáticas foram observadas com alteração da direção do gradiente espacial de precipitação e umidade relativa, devido aos efeitos da área desmatada;

A elevação na densidade de mosquitos coletados na Flona de Caxiuanã pode estar relacionado com as variações atmosféricas locais na escala de tempo do que com a escala de clima. As contribuições deste estudo são de grande importância para o entendimento dos efeitos das mudanças climáticas e a sua influência na biodiversidade, no monitoramento ambiental e no planejamento de políticas públicas.

\section{REFERÊNCIAS BILBIOGRÁFICAS}

CARVAlHo, T., MAGAlHAES, A. S.; DOMingueS, E. D. Desmatamento e a contribuição econômica da floresta na Amazônia. Estud. Econ., 2016. São Paulo, vol.46, n.2, p. 499-531, abr-jun.

COHEN, J. C. P.; DIAS, M. A. F. S. ; NOBRE, C. A. Environmental Conditions Associated with Amazonian Squall Lines: a case study. Monthly Weather Review, JCR, 1995, v. 123, p. 3163-3174.

CONFALONIERI, U. E. C.; NETO, C. C. Diversity of Mosquito Vectors (Diptera: Culicidae) in Caxiuanã, Pará, Brazil. Interdisciplinary Perspectives on Infectious Diseases, 2012. Volume 2012, Article ID 741273, 8 pages, doi: $10.1155 / 2012 / 741273$.

CHOU, C.; CHIANG, J. C. H.; LAN, C.; CHUNG, C.; LIAO, Y.; LEE, C. Increase in the range between wet and dry season precipitation. Nature Geoscience. 2013. Vol 6, DOI:10.1038/NGEO1744.

FIGUEROA, S. N.; NOBRE, C. A. Precipitions distribution over central and western tropical South America. Climanálise - Boletim de monitoramento e análise climática, 1990. 5(6): 36-45.

FISH, G. et al. Uma revisão geral sobre o clima da Amazônia. Acta Amazonica, 1998. 28(2): p.101-126.

FORATTINI, O. P. Culicidologia Médica. Identificação, biologia, epidemiologia. Edusp. 2002. São Paulo. vol. 2: 860pp.

GONZALEZ, R. A.; ANDREOLI, R. V.; CANDIDO, L. A.; KAYANO, M. T.; SOUZA, R. A. F. A influência do evento El Niño - Oscilação Sul e Atlântico Equatorial na precipitação sobre as regiões norte e nordeste da América do Sul. Acta Amazônica. 2013. VOL. 43(4), 469 - 480.

HASTENRATH, S.; HELLER, L. Dynamics of climatic hazards in Northeast Brazil. Quarterly Journal of the Royal Meteorological Society. 1977. v. 103, n. 435, p.77-92, Jan.

HOPP, M. J.; FOLEY, J. A. Worldwide fluctuations in dengue fever cases related to climate variability. Clim Res. 2003; 25(1):85-94. 
LORENZ, C.; AZEVEDO, T. S.; VIRGINIO, F.; AGUIAR, B. S.; CHIARAVALLOTINETO, F.; SUESDEK, L. Impact of environmental factors on neglected emerging arboviral diseases. PLoS, Neglected Tropical Disease, 2017, 11(9):e0005959. doi.org/10.1371/journal.pntd.0005959.

MARENGO, J. Interannual variability of surface climate in the Amazon basin. International Journal of Climatology, 1992. 12(8), 853-863.

MARENGO, J. A.; NOBRE, C. A.; TOMASELLA, J. The Drought of Amazonia in 2005. American Meteorological Society, 2008. vol. 21, p. 495-516, DOI: 10.1175/2007JCLI1600.1.

\section{L12703 1 of5}

MARENGO J. A.; TOMSASELLA, J.; ALVES, L. M.; SOARES, W.; RODRIGUEZ, D. A. The drought of 2010 in the context of historical droughts in the Amazon region. Geophysical Research Letters. JCR, 2011, v. 38, p. 1-5.

MELLO, Y. R.; KOHLS, W.; OLIVEIRA, T. M. N. Uso de diferentes métodos para o preenchimento de falhas em estações pluviométricas. Boletim de geografia, 2017, Maringá, v. 35, n. 1, p. 112-121.

MOHR, K. I.; ZIPSER, E. J. Mesoscale convective systems defined by their 85$\mathrm{GHz}$ ice scattering signature: size and intensity comparison over tropical oceans and continents. Monthly Weather Review. 1996. Vol. 124, pp. 2416-2437, DOI: 10.1175/1520-0493(1996)124<2417: MCSDBT>2.0.CO;-2

NEPSTAD, D. C.; MOREIRA, A. G.; ALENCAR, A. A. A floresta em chamas: Origens, impactos e prevenção do fogo na Amazônia. Programa piloto para a proteção das florestas tropicais do Brasil. 1999. Brasília. 202.p;il. ISBN: 8587827-07-3.

NOBRE, A. D. O futuro climático da Amazônia, Relatório de avaliação científica. ARA - Articulación Regional Amazônica. 2014.

NOBRE, P.; SHUKLA, J. Variations of sea surface temperature, wind stress and rainfall over the tropical Atlantic and South America. Journal of Climate. 1996. Vol. 9, p.2464-2479. DOI: 10.1175/15200442(1996)009<2464:VOSSTW>2.0.CO;2.

PATZ, J. A.; MARTENS, W.J.M.; FOCKS, D. A.; et al. Dengue fever epidemic potential as projected by general circulation models of global climate change. Environ Health Perspect. 1998; 106(3):147-53.

PINTO, C. S.; CONFALONIERI, U. E. C.; MASCARENHAS, B. M. "Ecology of Haemagogussp. and Sabethe ssp. (Diptera: Culicidae) in relation to the microclimates of the Caxiuanã National Forest, Pará, Brazil, "Memorias do Instituto Oswaldo Cruz, 2009. vol. 104, no. 4, pp. 592-598.

PINTO, C. S. Ecologia de culicídios vetores de febre amarela silvestre em relação ao microclima na floresta nacional de Caxiuanã-Melgaço-PA. Dissertação de Mestrado em Zoologia, 2007. MPEG, Belém-PA.

RAO, V. B.; CAVALCANTI, I. F. A.; HADA, K. Annual variation of rainfall over Brazil and water vapor characteristics over South America. Journal of Geophysical Research. 1996, vol. 101. N D21, pp. 26539-26551. 
SANTOS, S. R. Q.; SILVA, R. B. C.; BARRETO, P. N.; NUNES, H. G. G. C.; RODRIGUES, R. S.; CAMPOS, T. L. O. B. Regime térmico e hídrico do solo para área de floresta tropical em anos de El Niño e La Niña, Caxiuanã-PA: Estudo de caso. Revista Brasileira de Meteorologia, 2011. v.26, n.3, 367 - 374.

TURNER, I. M. The ecology of trees in the tropical rain forest. Cambridge University Press, Cambridge. 2001.

WWF. Amazon Rainforest, Amazon Plants, Amazon River Animals. Consultado em 10 de outubro de 2017. 